\title{
A Low Complexity Detector for Downlink SCMA Systems
}

\author{
Lining Tian ${ }^{1}$, Minjian Zhao ${ }^{2}$, Jie Zhong ${ }^{3}$, Pei Xiao ${ }^{4}$, Lei Wen ${ }^{5}$
}

\begin{abstract}
Sparse Code Multiple Access (SCMA) is a novel non-orthogonal multiple access scheme for 5G systems, in which the logarithm domain message passing algorithm (Log-MPA) is applied at the receiver to achieve near-optimum performance. However, the computational complexity of Log-MPA detector is still a big challenge for practical implementation, especially for energysensitive user equipments in the downlink scenario. In this paper, a Region-Restricted detector with an improved Log-MPA (RRL detector) is proposed for downlink SCMA systems, in which the complexity is reduced from two perspectives. To avoid unnecessary calculations when searching the superposition constellation exhaustively, the proposed RRL detector updates the function nodes only within a restricted search region. While constellation points outside the search region are neglected, the performance is well maintained which is verified by simulations. Besides, the original Log-MPA heavily relies on exponential operations, resulting in high computational complexity. To solve this problem, an improved Log-MPA is also put forward in this paper to make a better compromise between complexity and performance. Simulation results show that the complexity of the RRL detector is reduced considerably while the bit error rate (BER) performance degrades unnoticeably.
\end{abstract}

\section{INTRODUCTION}

With the development of mobile devices and Internet of Things, explosive traffic growth and rapidly increased requirements are expected in the fifth generation $(5 \mathrm{G})$ wireless communications. To support massive connectivity with a large number of devices, multiple access technology is undergoing a paradigm shift from orthogonal to non-orthogonal based approaches [1].

Sparse code multiple access (SCMA) [2] is a novel non-orthogonal multiple access technique which derives from low density signature (LDS) [3] [4]. Moreover, SCMA replaces QAM modulation and LDS spreading with multi-dimensional codebooks, enabling SCMA to reap shaping gains. Due to the sparse structure of SCMA, the message passing algorithm (MPA) can be efficiently applied to approximate the optimum Maximum Likelihood (ML) detector. To reduce the complexity of the MPA detector, [1] proposed a Max-Log-MPA in which MPA is implemented in logarithm domain and the Jacobian logarithm max $^{*}$ is simplified as maximization operation max. However, the simplification results in additional performance degradation. [5] [6] investigated several better performing Log-MPA detectors by replacing max $^{*}$ with approximate functions max. However, this method brings extra multiplications and additions. [7] proposed a fixed low complexity detector based on partial marginalization. In [8], a shuffled MPA detection scheme for SCMA systems is proposed. In [9], the complexity of SCMA detector is reduced by an edge selection approach, where adaptive Gaussian approximation is applied to unselected edges. [10] proposed several techniques to reduce the SCMA detection, including codebook design with a specific structure and detection techniques taking advantage of the codebook structure. [11] investigated a low-complexity decoding algorithm based on list sphere decoding. However, most of these prior works [7]- [11] are for uplink channels, which means the proposed detectors are not suitable for downlink SCMA systems. This is due to the distinct channel environment in downlink systems, where symbols collided at the same resource are going through the same channel, which makes edge selection algorithm [9] and techniques in [10] noneffective. Other detectors produce unexpected performance loss when applied in downlink systems.

Motivated by this, we concentrate on the design of SCMA detector in downlink channels in this paper. The computational complexity of SCMA detector is mainly governed by the complexity of operations at function nodes (FNs) as well as the number of $\exp (\cdot)$ calculations [10]. To tackle these problems, we propose a novel Region-Restricted detector with an improved LogMPA (RRL detector) for downlink SCMA systems in this paper. The proposed RRL detector reduces the complexity effectively from two different perspectives. In the RRL detector, building on our previous work [12], the superposition constellation of SCMA is partitioned by several rays and concentric circles. Then the received signal falls within one of the regions bounded by the rays and circles (denoted by $\mathcal{D}$ ). Based on $\mathcal{D}$, a search region $\mathcal{S}$ is obtained subsequently which is larger than $\mathcal{D}$. Instead

L. Tian, M. Zhao, J. Zhong are with the College of Information Science and Electronic Engineering, Zhejiang University, Hangzhou 310027, China (E-mail: ${ }^{1}$ tianlining@zju.edu.cn; ${ }^{2}$ mjzhao@zju.edu.cn; ${ }^{3}$ zhongjie@zju.edu.cn). P. Xiao is with the Institute for Communications Systems Department of Electronic Engineering, University of Surrey, Guildford, Surrey, GU2 7XH, United Kingdom (E-mail: ${ }^{4}$ p.xiao@ surrey.ac.uk). L. Wen is with the College of Electronical Science and Engineering, National University of Defence Technology, Changsha 410073, China (E-mail: ${ }^{5}$ l.wen@ surrey.ac.uk)

This work is supported by the National Science Foundation of China (NSFC) under Grant 91538103, and the Fundamental Research Funds for the Central Universities. 


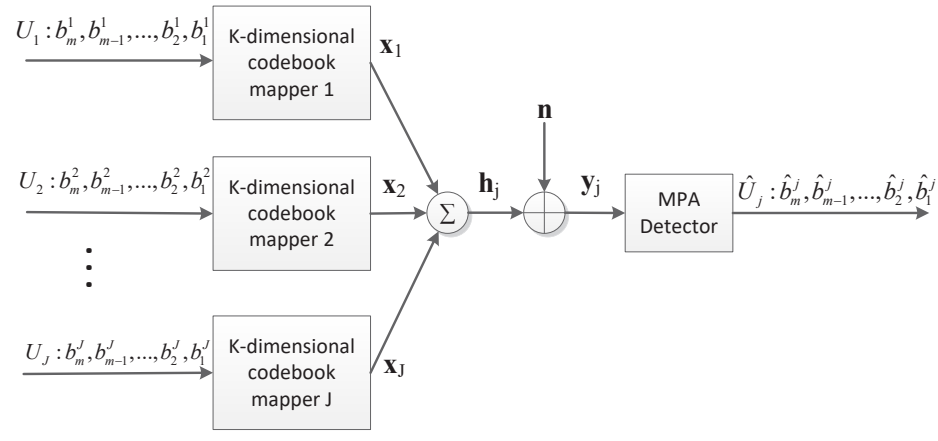

Fig. 1. SCMA downlink system between the transmitter and the $j$-th receiver.

of searching the whole constellation exhaustively, only constellation points in $\mathcal{S}$ are searched in each iteration process while the other points are neglected. To further reduce the complexity, in the RRL detector, the Log-MPA is improved by combining $\max / \tilde{m a x}$ operations properly to obtain a better trade-off between complexity and performance. Our simulation results show that the RRL detector performs close to the original Log-MPA detector while the complexity is reduced remarkably, especially for additions and exponential operations.

The rest of this paper is organized as follows. Section II introduces the downlink SCMA system model along with the original Log-MPA detector. In Section III, the proposed RRL detector is presented. Section IV compares the BER performance and complexity between the RRL and original detector. Finally, conclusions are drawn in Section V.

Notations: We use bold upper case letters to denote matrices, bold lower case letters to denote vectors and lower case letters to denote scalars. The notations $(\cdot)^{H}$ and $(\cdot)^{T}$ respectively denote the Hermitian transpose and transpose. Also, $\operatorname{diag}(\boldsymbol{h})$ denotes a diagonal matrix formed by the elements of vector $\boldsymbol{h}$, and $\operatorname{real}(h)$ and $\operatorname{imag}(h)$ respectively stand for the real and imaginary part of $h$. The maximum of $(\cdot)$ is denoted as $\max (\cdot)$.

\section{SYSTEM MODEL}

\section{A. Downlink SCMA system}

Consider a downlink channel where a base station (BS) is communicating with $J$ users, which is depicted in Fig. 1. For each user, $m=\log _{2}(M)$ bits are transmitted by a $K$-dimensional complex codebook with $M$ codewords, and the $K$ dimensional codebook has only $N$ nonzero elements, where $N \ll K$. For simplicity, the codeword of user $j$ is denoted as $\boldsymbol{x}_{j}=\left[x_{1}^{j}, x_{2}^{j}, \ldots, x_{K}^{j}\right]^{T} \in \mathcal{X}_{j}$, and the $K$-dimensional $M$-sized $\mathcal{X}_{j}$ can be constructed by algorithms proposed in [13]. Then $J$ codewords are superposed at the transmitter, and the received signal at user $j$ can be expressed as

$$
\boldsymbol{y}^{j}=\operatorname{diag}\left(\boldsymbol{h}^{j}\right) \sum_{j=1}^{J} \boldsymbol{x}_{j}+\boldsymbol{n},
$$

where $\boldsymbol{h}^{j}=\left[h_{1}^{j}, h_{2}^{j}, \ldots, h_{K}^{j}\right]^{T}$ is the channel gain between the transmitter and user $j$, and $\boldsymbol{n} \sim \mathcal{C N}\left(\mathbf{0}, \delta^{2} \boldsymbol{I}\right)$ denotes the Gaussian noise, where $\boldsymbol{I}$ is the identity matrix. For simplicity, the superscripts of $\boldsymbol{y}^{j}$ and $\boldsymbol{h}^{j}$ in (1) are removed from the equation, and thus the received signal at the $k$-th resource can be written as

$$
y_{k}=h_{k} \sum_{j=1}^{J} x_{k}^{j}+n_{k}, k=1,2, \ldots, K .
$$

Commonly, $\boldsymbol{X}=\left[\boldsymbol{x}_{1}, \boldsymbol{x}_{2}, \ldots, \boldsymbol{x}_{K}\right]$ is called SCMA matrix with $d_{f}$ non-zero elements in each row and $N$ non-zero elements in each column. Now define $\xi_{k}$ and $\zeta_{j}$ as the subsets of indices corresponding to the non-zero locations in the $k$-th row and the $j$-th column of SCMA matrix, respectively, i.e., $\xi_{k} \triangleq\left\{j \in\{1,2, \ldots, J\} \mid x_{k}^{j} \neq 0\right\}$ and $\zeta_{j} \triangleq\left\{k \in\{1,2, \ldots, K\} \mid x_{k}^{j} \neq 0\right\}$. Then (2) can be rewritten as

$$
y_{k}=h_{k} \sum_{j \in \xi_{k}} x_{k}^{j}+n_{k} \triangleq h_{k} z_{k}+n_{k},
$$

where $z_{k}=\sum_{j \in \xi_{k}} x_{k}^{j} \in \mathcal{Z}_{k}$, and $z_{k}$ can be considered as a symbol superposed by $d_{f}$ symbols from different users. Just as mentioned in [12], the constellation $\mathcal{Z}_{k}$ is referred to as superposition constellation. Obviously, the size of $\mathcal{Z}_{k}$ is $\left|\mathcal{Z}_{k}\right|=M^{d_{f}}$ which increases exponentially with $d_{f}$. 


\section{B. The original Log-MPA detector}

Due to the sparse property of SCMA matrix, the Log-MPA can be applied at the receiver to reduce the complexity while maintaining desired performance. Let $L_{j \rightarrow k}^{t}\left(\boldsymbol{x}_{j}\right)$ and $L_{k \rightarrow j}^{t}\left(\boldsymbol{x}_{j}\right)$ be the messages sent from user node (UN) $j$ and function node (FN) $k$ along edge $e_{j, k}$ at the $t$-th iteration. For $z_{k} \in \mathcal{Z}_{k}$, the probability of $z_{k}$ in logarithm domain after observing $y_{k}$ can be measured by

$$
M\left(z_{k}\right)=-\frac{1}{2 \sigma^{2}}\left|y_{k}-h_{k} z_{k}\right|^{2},
$$

then the $k$-th FN can be updated as below

$$
L_{k \rightarrow j}^{t}\left(\boldsymbol{x}_{j}\right)=\lambda \max _{z_{k} \in \mathcal{Z}_{k}: \boldsymbol{x}_{j}}\left(\sum_{j^{\prime} \in \xi_{k} \backslash j} L_{j^{\prime} \rightarrow k}^{t-1}\left(\boldsymbol{x}_{j^{\prime}}\right)+M\left(z_{k}\right)\right),
$$

where $\lambda$ denotes the normalization coefficient [14] and $\max ^{*}$ is the Jacobian logarithm

$$
\begin{gathered}
\max ^{*}(a, b)=\log \left(e^{a}+e^{b}\right)=\max (a, b)+C(a-b), \\
C(x)=\log \left(1+e^{-|x|}\right),
\end{gathered}
$$

with $C(x)$ represents the correction term.

The $j$-th UN is updated by the extrinsic information from connected FNs:

$$
L_{j \rightarrow k}^{t}\left(\boldsymbol{x}_{j}\right)=\sum_{k^{\prime} \in \zeta_{j} \backslash k} L_{k^{\prime} \rightarrow j}^{t}\left(\boldsymbol{x}_{j}\right) .
$$

After the message passing algorithm has converged or has reached the maximum number of iterations $t_{\max }$, the estimated value of user node $j$ is given by

$$
\hat{\boldsymbol{x}}_{j}=\arg \max _{\boldsymbol{x}_{j} \in \mathcal{X}_{j}} \sum_{k \in \zeta_{j}} L_{k \rightarrow j}^{t}\left(\boldsymbol{x}_{j}\right)
$$

\section{PRoposed RRL Detector}

The complexity of the original Log-MPA detector is dominated by (5), since it performs axa $^{*}$ operation by $M^{d_{f}-1}-1$ times for each $\boldsymbol{x}_{j} \in \mathcal{X}_{j}$. In general, all constellation points in $\mathcal{Z}_{k}$ are involved in the calculations of (4)-(7), which cannot be sustained for energy-sensitive user equipments. To address this issue, we propose a novel RRL detector where constellation points in a specific search region are searched to compute (5). Besides, in our proposed RRL detector, the Log-MPA is improved by applying an appropriate combination of $\max / \tilde{m a x}$ operations to avoid $\exp (\cdot)$ calculations.

\section{A. RR-MPA for AWGN channels}

For simplicity, consider AWGN channels firstly, i.e., $h_{k}=1, k=1,2, \ldots, K$. In (5), $\sum_{j^{\prime} \in \xi_{k} \backslash j} L_{j^{\prime} \rightarrow k}^{t-1}\left(\boldsymbol{x}_{j^{\prime}}\right)$ is the extrinsic information from connected user nodes, and $M\left(z_{k}\right)$ is proportional to the Euclidean distance between $y_{k}$ and $z_{k}$. Just as we have defined, $z_{k}$ is a point from the superposition constellation. For a point $z_{k}$ who is far away from $y_{k}, M\left(z_{k}\right)$ is so small that the addition $\sum_{j^{\prime} \in \xi_{k} \backslash j} L_{j^{\prime} \rightarrow k}^{t-1}\left(\boldsymbol{x}_{j^{\prime}}\right)+M\left(z_{k}\right)$ makes little contribution to $L_{k \rightarrow j}^{t}\left(\boldsymbol{x}_{j}\right)$ whatever the extrinsic information is. In this case, calculating (5) is a waste of computation. In our proposed RR-MPA, constellation points to calculate (5) are selected elaborately.

In SCMA systems, the superposition constellation is quite different from that in the optimized LDS systems [12]. For example, the superposition constellations with $M=4$ and $M=16$ are given in Fig. 2, where rotated QPSK and 16-QAM constellations respectively are employed by each user in each dimension. We can see from the figure that points close to the origin are clustered densely, and points far away from the origin are distributed sparsely. The low-complexity detector in [12] can not be applied in SCMA systems. Based on this, we put forward the RR-MPA which is described in detail below.

Firstly, the superposition constellation is partitioned by several rays and concentric circles just as depicted in Fig. 3 (a). Eight rays shooting from the origin (including the real and imaginary axis) $l_{0}, l_{1}, \ldots, l_{7}$ divide the constellation into 8 sectors with central angles equal $\pi / 4$. In radial direction, $P$ concentric circles with radius $r_{0}, r_{1}, \ldots, r_{P}$ partition the constellation into several annuluses. Finally, we get three kind of shaped regions bounded by these rays and concentric circles, marked by the shaded areas in Fig. 3 (a). Just as we have defined, the bounded region containing the the received signal $y_{k}$ is referred to as the decision region $\mathcal{D}$ and it can be determined by the magnitude and the phase of $y_{k}$ which are denoted as $\left|y_{k}\right|$ and $\theta_{y k}$, respectively. 


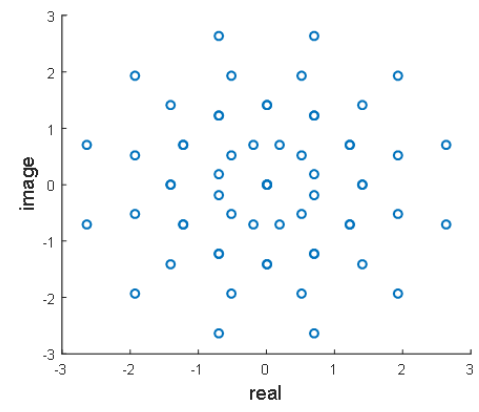

(a)

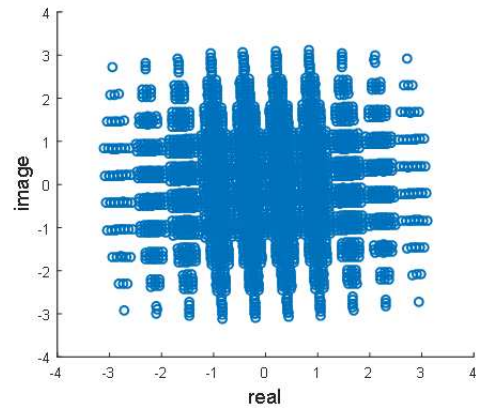

(b)

Fig. 2. (a). The superposition constellation of SCMA systems with $M=4$ and $d_{f}=3$; (b). The superposition constellation of SCMA systems with $M=16$ and $d_{f}=3$.

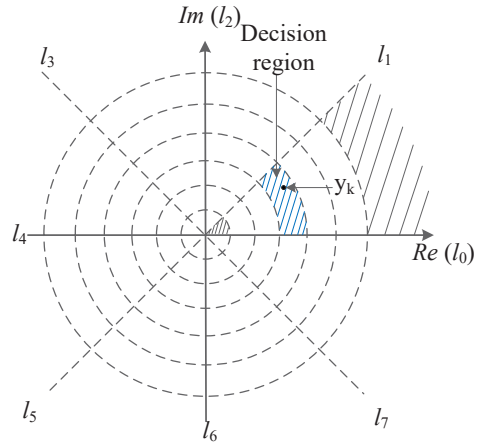

(a)

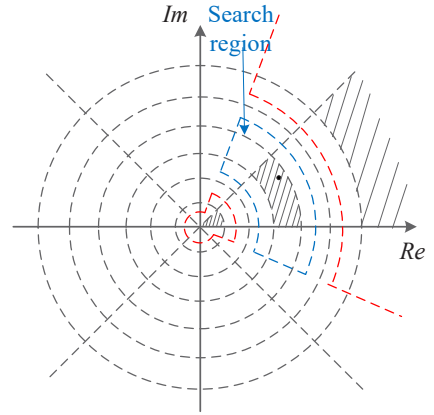

(b)

Fig. 3. (a). The decision region is the bounded region containing the received signal; (b). The search region is determined based on $\mathcal{D}$.

In general, constellation points in $\mathcal{D}$ are closer to $y_{k}$, so only these points are searched when making hard decision. However, it is inappropriate for MPA since FNs are passing soft decision messages to connected UNs. For example, it is not advisable to search $\mathcal{D}$ only when $y_{k}$ is on the boundary of $\mathcal{D}$. Thus, the region within which constellation points are searched and calculated should be larger than $\mathcal{D}$, and we refer to it as search region $\mathcal{S}$, shown in Fig. 3 (b). We observe that $\mathcal{S}$ contains $\mathcal{D}$, which means points not only in $\mathcal{D}$ but also around $\mathcal{D}$ are searched and hence involved in the calculation of (5), whereas points outside $\mathcal{S}$ are ignored due to their far distances to $y_{k}$. The search region $\mathcal{S}$ can be determined based on $\mathcal{D}$ as follows.

For simplicity, just as depicted in Fig. 4 (a), the diameter and central angle of $\mathcal{D}$ and $\mathcal{S}$ are denoted as $r_{D}, \theta_{D}, r_{S}$ and $\theta_{S}$, respectively. Let $r_{S}=\beta r_{D}, \theta_{S}=\alpha \theta_{D}$, where $\beta \geq 1$ and $\alpha \geq 1$ are two coefficients. Let $r_{i}=i r_{0}$ for all $i=1,2, \ldots, P$, which

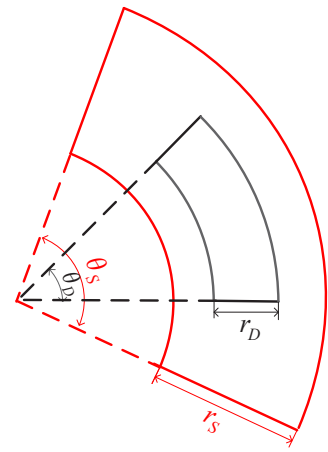

(a)

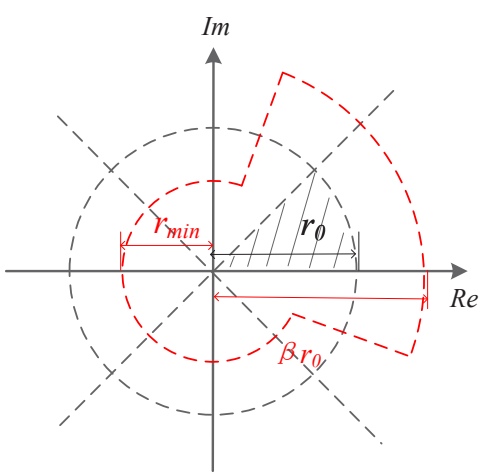

(b)

Fig. 4. (a). The diameter and central angle of $\mathcal{D}$ and $\mathcal{S}$; (b). The special case of $\mathcal{S}$. 
TABLE I

THE COMPLEXITY OF FUNCTION NODES OF THE DETECTORS IN III-C.

\begin{tabular}{ccccc}
\hline & max operations & exponential operations & additions & multiplications \\
\hline the original Log-MPA detector & $M J\left(M^{d_{f}-1}-1\right)$ & $2 M J\left(M^{d_{f}-1}-1\right)$ & $3 M J\left(M^{d_{f}-1}-1\right)+D_{1}$ & $D_{2}$ \\
the Max-Log-MPA detector & $M J\left(M^{d_{f}-1}-1\right)$ & 0 & $D_{1}$ & $D_{2}$ \\
the detector in [5] & $M J\left(M^{d_{f}-1}-1\right)$ & 0 & $4 M J\left(M^{d_{f}-1}-1\right)+D_{1}$ & $M J\left(M^{d_{f}-1}-1\right)+D_{2}$ \\
the improved Log-MPA detector & $M J\left(M^{d_{f}-1}-1\right)$ & 0 & $4 M J+D_{1}$ & $M J+D_{2}$ \\
\hline
\end{tabular}

is verified reasonable by simulation results later.

Suppose $\mathcal{D}$ is bounded by rays $l_{i}, l_{(i+1) m o d 8}$, then the phase of constellation point $z_{k}$ in $\mathcal{S}$ should be satisfied with

$$
\theta_{l_{i}}-\frac{(\alpha-1) \pi}{8}<\theta_{z k}<\theta_{l_{(i+1) \bmod 8}}+\frac{(\alpha-1) \pi}{8},
$$

where $\theta_{l_{i}}, \theta_{l_{(i+1) \bmod 8}}, \theta_{z k}$ are the phases of $l_{i}, l_{(i+1) \bmod 8}$ and $z_{k}$, respectively.

If $\mathcal{D}$ is sector-shaped which is bounded by $r_{0}$, the magnitude of $z_{k} \in \mathcal{S}$ satisfies

$$
\left|z_{k}\right|<\beta r_{0} .
$$

For annular-sector-shaped $\mathcal{D}$ which is bounded by $r_{j}$ and $r_{j+1}, j=0,1, \ldots, P-1$, the magnitude of $z_{k} \in \mathcal{S}$ satisfies

$$
(j-1) r_{0}-\frac{(\beta-1) r_{0}}{2}<\left|z_{k}\right|<j r_{0}+\frac{(\beta-1) r_{0}}{2} .
$$

For the last kind of shaped $\mathcal{D}$ which is bounded by $r_{P}$, point $z_{k}$ in $\mathcal{S}$ satisfies

$$
\left|z_{k}\right|>\operatorname{Pr}_{0}-\frac{(\beta-1) r_{0}}{2} \text {. }
$$

As a result, points in $\mathcal{S}$ can be selected by (10) and (11)-(13), and then $\mathcal{S}$ is determined directly on the basis of $\mathcal{D}$. It is also worth pointing out that there is a special case which is shown in Fig. 4 (b). For sector-shaped $\mathcal{D}, y_{k}$ is close to the origin. In this case, the Euclidean distance between two points belonging to different sectors is not large enough. Thus, $z_{k}$ has a close distance to $y_{k}$ even though $z_{k}$ does not satisfy (10). To solve the problem, for sector-shaped $\mathcal{D}$, constellation points $\left|z_{k}\right|<r_{\min }$ should also be considered, regardless of what $\theta_{z k}$ is. We can see that the search region $\mathcal{S}$ is the union of a disk and a sector. For other kind of $\mathcal{D}, \mathcal{S}$ has the same shape as $\mathcal{D}$ with a proper increase on the boundary.

Note that the RR-MPA is similar to the list sphere decoding based MPA [11], which restricts the search region to a hypersphere. Nevertheless, our proposal has lower complexity and is simpler to implement because the search region $\mathcal{S}$ can be calculated based on $\mathcal{D}$ off-line according to Equ. (10)-(13), which means the calculation of $\mathcal{S}$ can be accomplished before the transmission. Therefore, in our RR-MPA detector, $\mathcal{S}$ can be determined directly after $\mathcal{D}$ is determined based on $y_{k}$ without any calculations. In contrast, the list sphere decoding based MPA wastes lots of computations and time to select the proper points to participate in the updating.

\section{B. RR-MPA for fading channels}

In downlink flat fading channels, the received signal at $k$-th resource is given by (3). To apply RR-MPA in fading channels, (3) is rewritten as

$$
\frac{y_{k}}{h_{k}}=z_{k}+\frac{n_{k}}{h_{k}}, k=1,2, \ldots, K .
$$

It can be also considered as the AWGN channel with received signal $y_{k} / h_{k}$ and noise $n_{k} / h_{k}$. Thus $y_{k} / h_{k}$ is used to determine the decision region $\mathcal{D}$ in the downlink fading channels. Note that it is difficult to recover signals when channels are in deep fading. Even worse, the RR-MPA scheme will increase the performance degradation severely. It is because of the Euclidean distances reduction caused by the deep fading, and the optimal points are usually not in $\mathcal{S}$ even though $\mathcal{S}$ is larger than $\mathcal{D}$. To avoid this condition, RR-MPA is applied only when $\left|h_{k}\right| \geq \gamma$ where $\gamma$ is a parameter. Otherwise (5) is calculated for all $z_{k} \in \mathcal{Z}_{k}$. As can be seen, larger $\gamma$ makes the detector perform better but with increased complexity, and vice versa. 


\section{The Improved Log-MPA}

The complexity of original Log-MPA detector is also governed by the number of $\exp (\cdot)$ calculations [10]. For illustration purposes, (5) is expressed simply as

$$
\log \sum_{i=1}^{n} e^{x_{i}}=f_{n}\left(x_{n}, f_{n-1}\left(x_{n-1}, \ldots, f_{2}\left(x_{2}, x_{1}\right)\right)\right),
$$

where $n=M^{d_{f}-1}$ and $x_{i}$ represents the addition $\sum_{j^{\prime} \in \xi_{k} \backslash j} L_{j^{\prime} \rightarrow k}^{t-1}\left(\boldsymbol{x}_{j^{\prime}}\right)+M\left(z_{k}\right)$. In the original Log-MPA detector, $f_{n}=$ $f_{n-1}=\ldots=f_{2}=\max ^{*}$, resulting in that exponential operations are performed by countless times.

To reduce the number of $\exp (\cdot)$ calculations, authors in [1] proposed a Max-Log-MPA detector in which the correction term $C(x)$ in (7) is neglected and the Jacobian logarithm is simplified as $\max ^{*}(a, b)=\max (a, b)$. Thus, in the Max-Log-MPA detector, (15) is calculated as

$$
\log \sum_{i=1}^{n} e^{x_{i}} \approx \max \left(x_{n}, \max \left(x_{n-1}, \ldots, \max \left(x_{2}, x_{1}\right)\right)\right) .
$$

The simplification eliminates the $\exp (\cdot)$ operations to achieve a complexity reduction, but at the cost of degraded performance. To tackle this problem, we propose an improved Log-MPA which exhibits a better trade-off between complexity and performance. For the sake of illustration, sort $\left\{x_{i}\right\}$ in descending order, namely $x_{1}^{d} \geq x_{2}^{d} \geq \ldots \geq x_{n}^{d}$, and (15) can be expressed as

$$
\log \sum_{i=1}^{n} e^{x_{i}}=\mathcal{F}_{n} \triangleq \max ^{*}\left(x_{n}^{d}, \max ^{*}\left(x_{n-1}^{d}, \ldots, \max ^{*}\left(x_{2}^{d}, x_{1}^{d}\right)\right)\right)=\max ^{*}\left(x_{n}^{d}, \mathcal{F}_{n-1}\right) .
$$

As $C(x)>0$ for all $x>0$, we have $\mathcal{F}_{n}>\mathcal{F}_{n-1}>x_{1}^{d} \geq \ldots \geq x_{n}^{d}$, and thus (17) can be expanded as

$$
\begin{aligned}
\mathcal{F}_{n} & =\mathcal{F}_{n-1}+C\left(\mathcal{F}_{n-1}-x_{n}^{d}\right) \\
& =\mathcal{F}_{n-2}+C\left(\mathcal{F}_{n-2}-x_{n-1}^{d}\right)+C\left(\mathcal{F}_{n-1}-x_{n}^{d}\right)=\ldots \\
& =\mathcal{F}_{1}+\underbrace{C\left(\mathcal{F}_{1}-x_{2}^{d}\right)+C\left(\mathcal{F}_{2}-x_{3}^{d}\right)+\ldots C\left(\mathcal{F}_{i-1}-x_{i}^{d}\right)+\ldots+C\left(\mathcal{F}_{n-1}-x_{n}^{d}\right)}_{\text {correction items }},
\end{aligned}
$$

where $\mathcal{F}_{1}=x_{1}^{d}$.

Since $x_{i}^{d} \geq x_{i+1}^{d}$ and $\mathcal{F}_{i}>\mathcal{F}_{i-1}$, we have $\mathcal{F}_{i}-x_{i+1}^{d}>\mathcal{F}_{i-1}-x_{i}^{d}$. We know that $C(x)$ is a decreasing function [5], and we get $C\left(\mathcal{F}_{i}-x_{i+1}^{d}\right)<C\left(\mathcal{F}_{i-1}-x_{i}^{d}\right)$ which means the correction items in (20) are decreasing with increasing $i$. In the Max-Log-MPA, all correction items are ignored which accounts for the performance loss.

To improve the performance, the first $Q$ correction items can be retained where $0 \leq Q \leq n-1$. However, sorting $\left\{x_{i}\right\}$ wastes plenty of time and computations. Inspired by [15], in which novel turbo decoding algorithms are proposed based on the application of $\max / \max ^{*}$ operations at different levels, in our proposed Log-MPA, we implement $\max ^{*}$ on $f_{n}$ while $f_{n-1}, \ldots, f_{2}$ are simplified as max, and thus $\mathcal{F}_{n} \approx \mathcal{F}_{1}+C\left(\mathcal{F}_{1}-x_{2}^{d}\right)$. Therefore, the largest correction item is retained and sorting operation is also avoided. While ignoring all other correction items, the performance is well maintained which is verified by simulations later.

To eliminate the exponential operation completely, $\max ^{*}$ is approximated by a non-linear function [5]

$$
C_{1}(x)=\left\{\begin{array}{l}
\frac{m}{n+x}+p \quad x \leq q, \\
0 \quad x>q
\end{array}\right.
$$

where $m=1.0807, n=1.1657, p=-0.1975, q=5$. Denote $\max (a, b)=\max (a, b)+C_{1}(a-b)$, and in our improved Log-MPA, (15) is computed as follows

$$
F \triangleq \tilde{\max }\left(x_{n}, \max \left(x_{n-1}, \ldots, \max \left(x_{2}, x_{1}\right)\right)\right) .
$$

Simulations and complexity investigations show that (22) provides a more favorable performance-complexity tradeoff.

To compare the complexities of the detectors mentioned above detailedly, the amount of computational operations of function nodes in one iteration are listed in TABLE. I, where $D_{1}$ and $D_{2}$ represent the additions and multiplications performed when the extrinsic information and $M\left(z_{k}\right)$ are involved. More precisely, $D_{1}=J M^{d_{f}}\left(2 d_{f}-1\right), D_{2}=4 M J$ and they are identical for all detectors. We can see from the table that the complexity of our improved Log-MPA is reduced significantly compared to the original detector and the detector in [5], while increased slightly compared to the Max-Log-MPA detector.

Performance comparisons are also given in Fig. 5. From the figure, it is observed that our proposed Log-MPA has a quite 


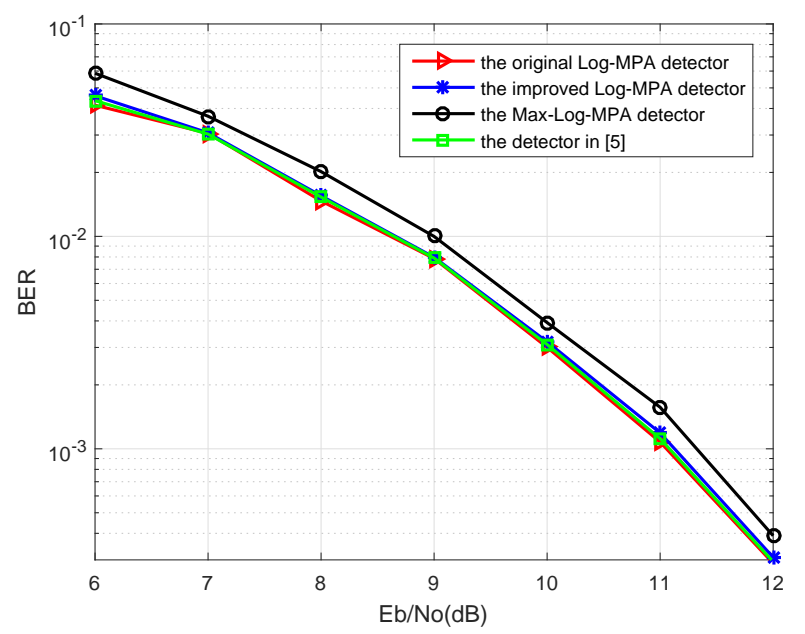

Fig. 5. Performance comparison between the detectors in III-C. The simulations are run with $J=6, K=4, M=4, t_{\max }=6$ over AWGN channels.

similar performance with the detector in [5], and both two are close to the original Log-MPA detector. While Max-Log-MPA has the lowest complexity, the performance is degraded by $0.5 \mathrm{~dB}$ when BER equals $10^{-3}$, compared with the other three detectors. From Fig. 5 and TABLE. I we can see that our proposed Log-MPA has the lowest complexity while the BER performance degrades unnoticeably.

\section{The RRL MPA detector}

By combining the above two techniques RR-MPA and the improved Log-MPA together, messages at FNs can be updated in a more simplified way:

$$
L_{k \rightarrow j}^{i}\left(\boldsymbol{x}_{j}\right)=\lambda \underset{z_{k} \in \mathcal{S}: \boldsymbol{x}_{j}}{F}\left(\sum_{j^{\prime} \in \xi_{k} \backslash j} L_{j^{\prime} \rightarrow k}^{t-1}\left(\boldsymbol{x}_{j^{\prime}}\right)+M\left(z_{k}\right)\right) .
$$

The novel RRL detector reduces the complexity considerably, and the detailed procedure of the proposed detector is provided in TABLE. II.

\section{SIMULATIONS}

In this section, the BER performance of downlink SCMA systems $\left(K=4, J=6, d_{f}=3, d_{c}=2, t_{\text {max }}=6\right)$ is evaluated over AWGN and Rayleigh fading channels. The SCMA matrix in [2] with $M=4$ and $M=16$ are considered. Besides, the average energy of user $j$ at $k$-th resource is set to 1 .

\section{A. Performance in AWGN channels}

Performance comparisons are shown in Fig. 6 and Fig. 7 for SCMA systems with $M=4$ and $M=16$, respectively. The number of constellation points in $\mathcal{S}$ is compared to that in $\mathcal{Z}_{k}$, and the ratio is also listed in the legend separated by a vertical bar, and the ratio can be used to represent the complexity ratio to some extent. From Fig. 6 it is observed that the proposed RRL detector with $r_{0}=1.5, \alpha=2.2$ performs almost as well as the original Log-MPA detector when $M=4$, but the complexity is reduced to $27.67 \%$. The complexity can be further decreased to $25.35 \%$ with performance degradation about $0.6 \mathrm{~dB}$ at $\mathrm{BER}=10^{-3}$. For the case with $M=16$, the complexity can be reduced to $17.59 \%$ with well-maintained performance when $\beta=1.5, \alpha=1.7$. The decrease of $\beta$ enables the RRL detector to reduce the complexity to $14.96 \%$ with $0.7 \mathrm{~dB}$ performance degradation at $\mathrm{BER}=10^{-3}$. In general, the larger $\alpha, \beta$ are, the more complex the proposed RRL detector is, and the better it performs.

\section{B. Performance in fading channels}

Fig. 8 and Fig. 9 compare the original detector and our proposed detector in downlink rayleigh fading channels. Based on IV-A, we choose $r_{0}=1.5, \alpha=2.2$ and $\beta=1.5, \alpha=1.7$ for $M=4$ and $M=16$, respectively. We can see from the figures that the proposed RRL detector with $\gamma=0$ performs poorly with about $8.0 \mathrm{~dB}$ and $2.5 \mathrm{~dB}$ degradation compared to the original detector at $\mathrm{BER}=10^{-3}$, for 4 -ary and 16-ary SCMA respectively. However, with a moderate increase of complexity, 
TABLE II

THE PROPOSED RRL DETECTOR
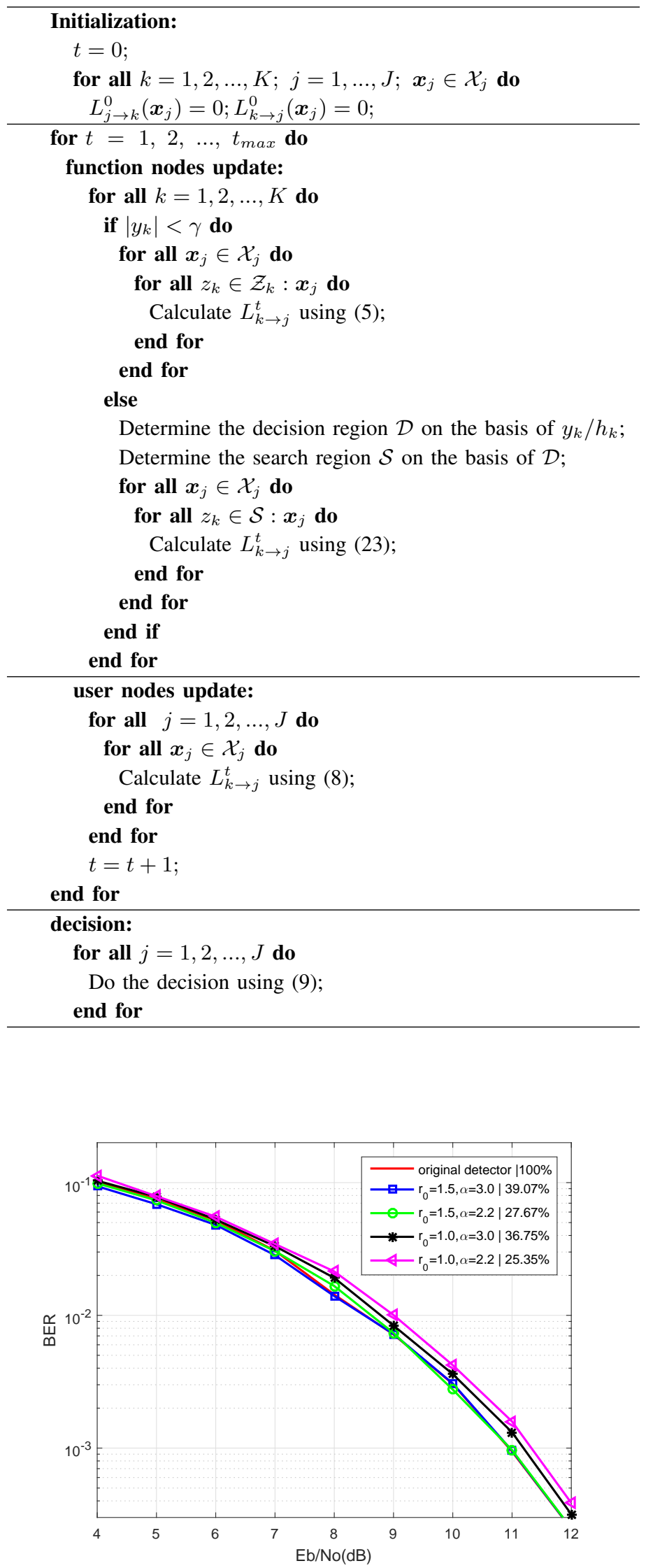

Fig. 6. BER performance comparison of SCMA detectors with $M=4$ in AWGN channels. $P=2, \beta=2.5, r_{m i n}=0.8$, and the RRL detectors with different $r_{0}, \alpha$ are investigated and compared. 


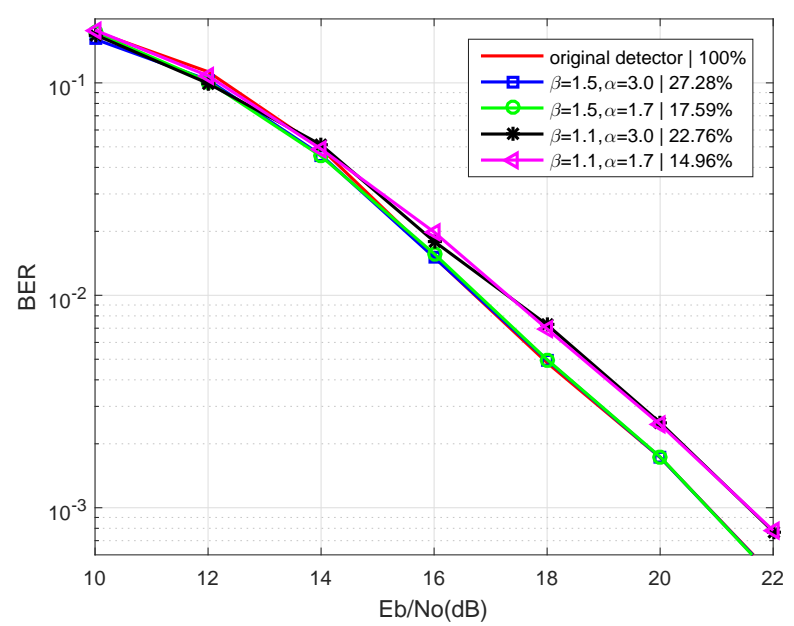

Fig. 7. BER performance comparison of SCMA detectors with $M=16$ in AWGN channels. $r_{0}=2, P=5, \beta=2.5, r_{\text {min }}=0.3$, and the RRL detectors with different $\beta, \alpha$ are investigated and compared.

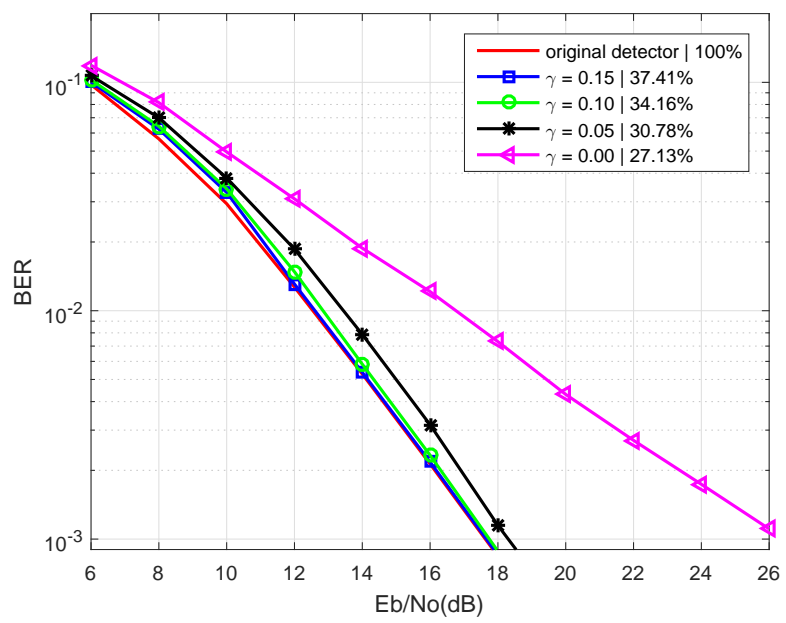

Fig. 8. BER performance comparison of SCMA detectors with $M=4$ in Rayleigh fading channels.

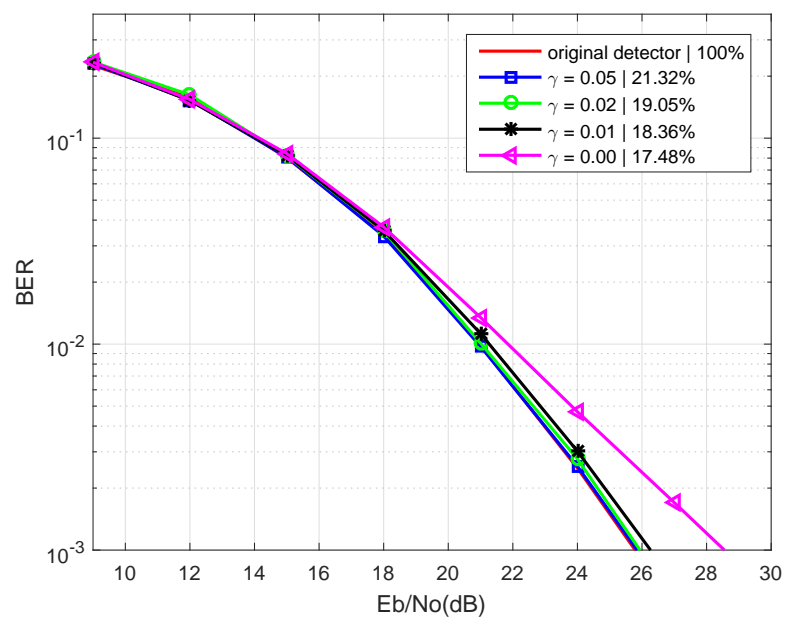

Fig. 9. BER performance comparison of SCMA detectors with $M=16$ in Rayleigh fading channels. 


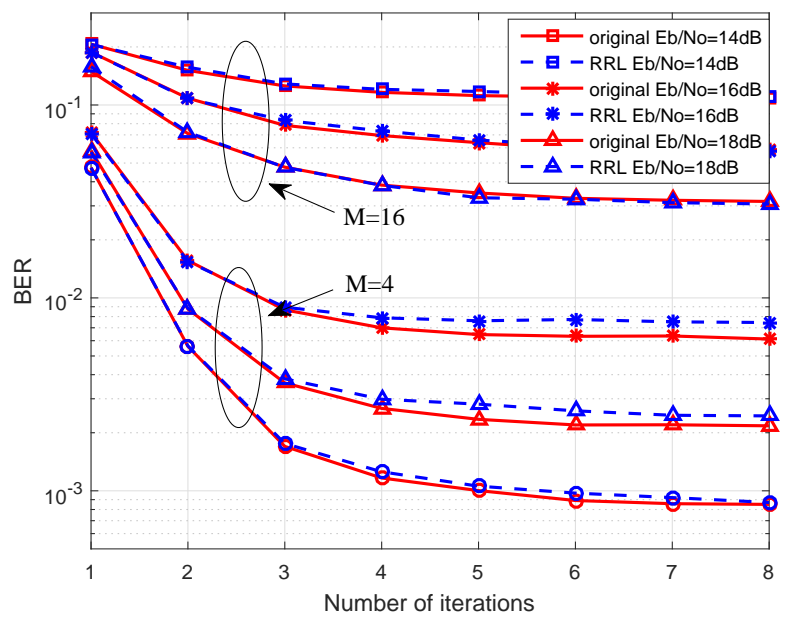

Fig. 10. Convergence behavior comparison between the original detect and the RRL detector.

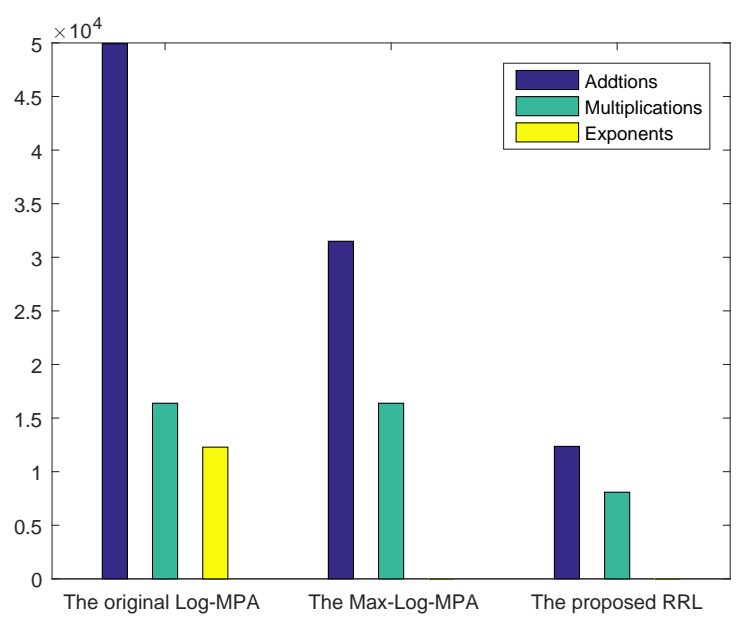

Fig. 11. Complexity comparison between the original Log-MPA, Max-Log-MPA and the RRL detector with $M=4$.

BER performance could be improved dramatically. Our proposed RRL detector reduces the complexity to $34.16 \%$ and $19.05 \%$ of the original detector for $M=4$ and $M=16$ while the performance is degraded unnoticeably. The complexity is increased slightly compared with the RRL detector in AWGN channels, and it is because an exhaustive search is implemented when the channel is in deep fading.

\section{Convergence behavior}

Fig. 10 shows the convergence behavior of the proposed detector and original detector at Eb/No $=14 \mathrm{~dB}, 16 \mathrm{~dB}, 18 \mathrm{~dB}$ in downlink rayleigh fading channels. We choose $\gamma=0.10(0.02)$ for $M=4(16)$, respectively. From the simulation results, the proposed detector exhibits the same convergence performance as the original detector, particularly for the first three iterations. After that, the proposed detector converges to a little higher BER compared to the original detector.

\section{Complexity analysis}

Fig. 11 and Fig. 12 illustrate the computational complexity of the proposed RRL detector, the Max-Log-MPA detector and the original Log-MPA detector in detail. The Max-Log-MPA reduces the amount of additions and eliminates the exponential operations by replacing $\max ^{*}$ with $\max$. As can be seen, the proposed RRL detector reduces the number of additions to about $26.0 \%$ and $18.8 \%$ when $M=4$ and $M=16$ respectively, compared with the original Log-MPA. The multiplications are not decreased as much as the additions because (21) introduces extra multiplications to eliminate the exponential operations. Besides, the RRL detector has lower complexity than the Max-Log-MPA and attains a better trade-off between the performance and computational complexity. 


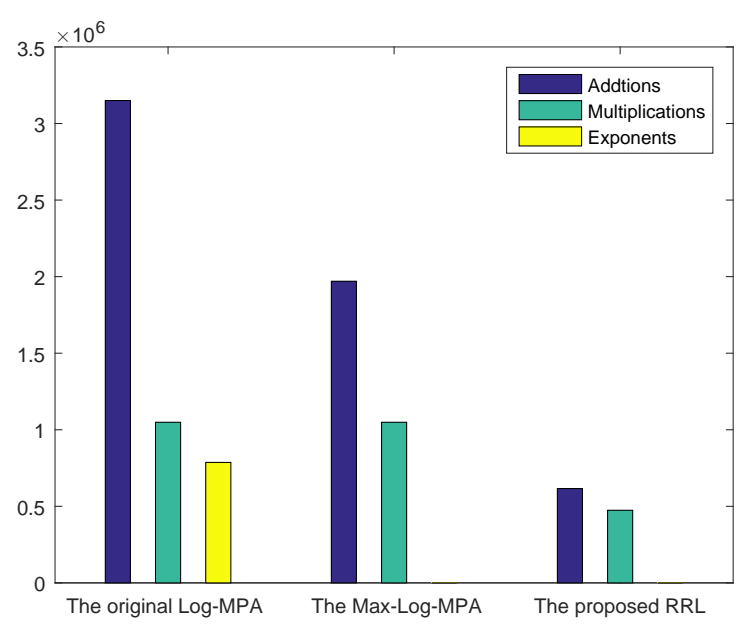

Fig. 12. Complexity comparison between the original Log-MPA, Max-Log-MPA and the RRL detector with $M=16$.

\section{CONCLUSION}

In this paper, we proposed a novel RRL detector for downlink SCMA systems. The proposed RRL detector substantially reduces the computational complexity by decreasing the number of constellation points involved in the updating of function nodes. In addition, the proposed RRL detector eliminates the exponential operations by applying an appropriate combination of $\max / \tilde{m a x}$ operations, offering performance close to the original detector. The RRL detector demonstrates a near-optimum BER performance with significantly reduced complexity.

\section{REFERENCES}

[1] Zhang, S., Xu, X., Lu, L., et al.: 'Sparse code multiple access: an engergy efficient uplink approach for 5G wireless systems,' Proc. IEEE Global Telecommun. Conf. (GLOBECOM), Austin, USA, Dec. 2014, pp. 4782-4787

[2] Nikopour, H., Baligh, H.: 'Sparse code multiple access,' Proc. IEEE Int. Symp. Personal Indoor and Mobile Radio Commun. (PIMRC), London, U. K., Sept. 2013, pp. 332-336

[3] Hoshyar, R., Wathan, F. P., Tafazolli, R.: 'Novel low-density signatures for synchronous CDMA systems over awgn channel,' IEEE Trans. Signal Process., 2008, 56, (4), pp. 1616-1626

[4] Wen, L., Razavi, R., Imran, M. A., et al.: 'Design of joint sparse graph for OFDM system,' IEEE Trans. on Wireless Commun., 2014, 14, (4), pp. $1823-1836$

[5] Xiao, K.,Xiao, B., Zhang, S., et al.: 'Simplified Multiuser detection for SCMA with sum-product algorithm,' Proc. Inter. Conf. on Wire. Commn. Signal Process. (WCSP), Nanjing, China, Oct. 2015, pp. 1-5

[6] Alizadeh, R., Savaria, Y.: 'Performance analysis of a reduced complexity SCMA decoder exploiting a low-complexity maximum-likelihood approximation,' Proc. Inter. Conf. on Elec. Circuits and Systems (ICECS), Monte Carlo, Dec. 2016, pp. 253-256

[7] Mu, H., Ma, Z., Alhaji, M., et al.: 'A fixed low complexity message pass detector for up-link SCMA system,' IEEE Wireless Commun. Lett., 2015, 4, (6), pp. 585-588

[8] Du, Y., Dong, B., Chen, Z., et al.: 'Shuffled multiuser detection schemes for uplink sparse code multiple access systems,' IEEE Commun. Lett., 2016, 20, (6), pp. 1231-1234

[9] Du, Y., Dong, B., Chen, Z., et al.: 'Low-complexity detector in sparse code multiple access sytems,' IEEE Commun. Lett., 2016, 20, (9), pp. 1812-1815

[10] Bayesteh, A., Nikopour, H., Taherzadeh, M., et al.: 'Low complexity techniques for SCMA detection,' Proc. IEEE Globecom Workshops (GC Wkshps), San Diego, USA, Dec. 2015, pp. 1-6

[11] Wei, F., Chen, W.: 'Low complexity Iterative receiver design for sparse code multiple access,' IEEE Trans. Commun., 2016, 65, (2), pp. 621-634

[12] Tian, L., Zhong, J., Zhao, M., et al.: 'An optimized superposition constellation and region-restricted MPA detector for LDS system,' Proc. Inter. Conf. on Commun. (ICC), Kuala Lumpur, Malaysia, May 2016, pp. 1-5

[13] Taherzadeh, M., Nikopour, H., Bayesteh, A., et al.: 'SCMA codebook design,' Proc. IEEE 80th Vehicular Technology Conference (VTC Fall), Vancouver, Canada, Sept. 2014, pp. 1-5

[14] Razavi, R., Al-Imari, M., Imran, M. A.,et al.: 'On recevier design for uplink low density signature OFDM(LDS-OFDM),' IEEE Trans. Commun., 2012, 60, (11), pp. 3499-3508

[15] Papaharalabos, S., Sweeney, P., Evans, B. G.,: 'SISO algorithms based on Max-Log-MAP and Log-MAP turbo decoding,' IET Commun., 2007, 1, (1), pp. $49-54$ 